\section{Re: Magnesium Intake and Depression in Adults}

To the Editor: We read with great interest the article by Tarleton and Littenberg ${ }^{1}$ on the relation between magnesium intake and depression in adults. We recognize the impact of depression in modern society, and we agree with the authors that current treatment options have their limitations. A possible role for magnesium could therefore be interesting. The authors found an association between low magnesium intake and depression among younger adults and a potential protective effect against depression in seniors. Although this sounds promising, we would like to discuss some methodologic issues about this study.

First, the authors chose to divide the range of magnesium intake within the study population into quantiles. This is remarkable, as the quintiles do not correspond to magnesium intake thresholds as defined by the estimated average requirements. ${ }^{2}$ The reason for the use of quantiles is not explained but would be of interest for the readers, since this choice has important consequences on the outcome: When the estimated average requirement definition for deficient magnesium intake is used, no association between magnesium intake and depression was found (adjusted relative risk $0.98 ; 95 \%$ confidence interval [CI], 0.88-1.07).

Second, based on 1-day dietary recall data, Tarleton and Littenberg ${ }^{1}$ found a deficient magnesium intake in $54 \%$ of the study population. Although this is a relatively high percentage, the authors stated in their discussion that the method of magnesium assessment was adequate. However, it is important to realize that the absorption of magnesium varies depending on the intake of magnesium. The absorption can vary between $30 \%$ and $40 \%$ in a normal diet and up to $80 \%$ with a low magnesium intake. ${ }^{3}$ Therefore, deficient magnesium intake cannot directly be translated into actual hypomagnesemia. From this perspective it is questionable whether the authors are really evaluating the magnesium status of their study group.

Third, when evaluating the multivariable adjusted analyses of low magnesium and depression, it is obvious that most variables have a much stronger association with depression than low magnesium intake (at most chronic kidney disease: odds ratio [OR], 2.50; 95\% CI, 1.663.79; and food insecurity: OR, 2.30; 95\% CI, 1.90-2.78). All those variables seem to be associated with socioeconomic status (SES) and lifestyle. Low magnesium intake may therefore be a proxy for an unhealthy lifestyle and/or a low SES. The association between SES and depression is well known: Low SES increases the risk of onset and persistence of depression. ${ }^{4}$ In addition, depression has been found to be associated with poor food consumption patterns, with the association probably being bidirectional. ${ }^{5,6}$

In conclusion, the idea that magnesium intake could modulate depression is interesting. Given the abovementioned concerns, however, we are doubtful about the described association between magnesium intake and depression. When further research is considered, we recommend first a prospective study instead of a randomized clinical trial.

Denise Sluimers, MS*

Nick L. Willemse, $\mathrm{MS}^{*}$

Marieke L.A. Landsmeer

Department of General Practice

Erasmus Medical Center

Rotterdam, The Netherlands

m.landsmeer@erasmusmc.nl

*These authors contributed equally to this letter.

\section{References}

1. Tarleton EK, Littenberg B. Magnesium intake and depression in adults. J Am Board Fam Med 2015;28:249-56.

2. Institute of Medicine (US) Standing Committee on the Scientific Evaluation of Dietary Reference Intakes. Dietary Reference Intakes for Calcium, Phosphorus, Magnesium, Vitamin D, and Fluoride. Washington, DC: National Academies Press; 1997. Available from: http://www.ncbi.nlm.nih.gov/ books/NBK109825/. Accessed July 27, 2015.

3. McLean RM. Magnesium and its therapeutic uses: a review. Am J Med 1994;96:63-76.

4. Lorant V, Croux C, Weich S, Deliege D, Mackenbach J, Ansseau M. Depression and socio-economic risk factors: 7-year longitudinal population study. Br J Psychiatry 2007; 190:293-8.

5. Akbaraly TN, Brunner EJ, Ferrie JE, Marmot MG, Kivimaki M, Singh-Manoux A. Dietary pattern and depressive symptoms in middle age. Br J Psychiatry 2009;195:408-13.

6. Anton SD, Miller PM. Do negative emotions predict alcohol consumption, saturated fat intake, and physical activity in older adults? Behav Modif 2005;29:677-88.

doi: 10.3122/jabfm.2015.05.150144

The above letter was referred to the author of the article in question, who offers the following reply.

\section{Response: Re: Magnesium Intake and Depression in Adults}

To the Editor: We thank Slumiers and colleagues ${ }^{1}$ for highlighting the challenges of interpreting outcomes from cross-sectional, population-based data. Preclinical and clinical studies indicate low magnesium intake may be associated with depressive symptoms. For instance, Singewald et $\mathrm{al}^{2}$ reported mice consuming a diet with very low magnesium content-consisting of only $10 \%$ of 
the daily requirement-showed depressive behavior. Therefore we chose to divide magnesium intake into quintiles to explore the possibility that an association might be seen only in those consuming much less than the estimated average requirement. Sluimers and colleagues are correct that dietary recall is not a perfect marker of hypomagnesemia. That the association is still significant despite the variation introduced by recall methods supports its robustness. Further, intake is amenable to intervention by patients, providers, and policymakers, making it an especially valuable target of analysis.

We agree that social and medical factors are stronger correlates of depression than magnesium intake, as Table 2 (p. 253) shows. We included these variables in the multivariate analysis to explore the possibility that they confound the association of magnesium intake and depression, as suggested by the correspondents. ${ }^{3}$ That magnesium intake remains significantly associated with depression in the models argues against confounding by these variables. Of course, residual confounding by unmeasured variables is a possibility, which is why prospective randomized trials are needed. We acknowledge that the relationship between magnesium and depression may be bidirectional, and we can only report an association. However, our study adds to the overall body of literature in support of exploring this relationship further. Finally, we see no virtue in a nonrandomized prospective trial because any such study would be subject to the same biases the authors point out in their letter.

Emily K. Tarleton, MS, RD

Benjamin Littenberg, MD

Center for Clinical and Translational Science

University of Vermont Burlington, VT

Emily.tarleton@uvm.edu

\section{References}

1. Sluimers D, Willemse NL, Landsmeer MLA. Re: magnesium intake and depression in adults. J Am Board Fam Med 2015;28:683-3.

2. Singewald N, Sinner C, Hetzenauer A, Sartori SB, Murck H. Magnesium-deficient diet alters depression- and anxiety-related behavior in mice-influence of desipramine and Hypericum perforatum extract. Neuropharmacology 2004;47: 1189-97.

3. Tarleton EK, Littenberg B. Magnesium intake and depression in adults. J Am Board Fam Med 2015;28:249-56.

doi: 10.3122/jabfm.2015.05.150194

\section{Re: The Use of Medical Scribes in Health Care Settings: A Systematic Review and Future Directions}

In the May/June issue of Fournal of the American Board of Family Medicine, Shultz and Holmstrom ${ }^{1}$ performed a systematic review of the literature investigating the effects of medical scribes on health care productivity, clinical quality, revenue, time efficiency, and patient-clinician interaction. We, at the University of California, San Francisco, Center for Excellence in Primary Care, applaud this research and agree that with the uptake of electronic health records in medical practices across the United States, much of the documentation and administrative burden has shifted to primary care providers. Using medical scribes to document visit notes and enter orders during the patient visit is a promising approach to improve the patient-clinician encounter in the current era of electronic health records.

Medical scribing can be embedded among other activities, and clinical staff members who perform the scribing function are not always called "scribes." The literature review by Shultz and Holmstrom ${ }^{1}$ therefore excluded a few relevant publications that did not have the word scribe in the title or abstract. To this end, we found 4 additional studies that explored the effect of medical scribes in the clinical setting. One study used a team-based care approach that paired each clinician with 2 clinical assistants who consistently work together to care for their patients. The clinical assistants took responsibility for many of the patient care tasks, and scribed during the physician encounter. Anderson and Halley ${ }^{2}$ found an increase physician productivity that resulted in increased income to offset the costs of the additional personnel and also improved patient and staff satisfaction. Another peer-reviewed study investigated the use of practice partners in 2 academic health center practices. Practice partners were also paired with physicians in a 2:1 ratio and performed scribing and other administrative functions, including assisting patients with the checkout process. Reuben et $\mathrm{al}^{3}$ found that the use of practice partners led to a reduction in physician time spent before and after sessions, shorter geriatric visits, and higher patient satisfaction.

In addition, 2 non-peer-reviewed studies of the use of medical scribes in primary care settings found improvements in clinician satisfaction; an increase in the accuracy of chart notes and productivity $^{4}$; a decrease in no-show rates, cycle time, and staff cost per relative value unit; an increase in gross net revenue per visit; and improvements in clinical quality measures. ${ }^{5}$ Based on the findings from Shultz and Holmstrom ${ }^{1}$ and the above additional literature, the variety of medical scribing and team documentation models can be divided in 2 broad categories: (1) the staff member accompanies the clinician during each patient visit and assists only with scribing and documentation, and (2) specially trained 